\title{
ROCK2 is a major regulator of axonal degeneration, neuronal death and axonal regeneration in the CNS
}

\author{
JC Koch ${ }^{\star, 1}$, L Tönges ${ }^{1}$, E Barski ${ }^{1}$, U Michel ${ }^{1}$, M Bähr ${ }^{1,2}$ and P Lingor ${ }^{1,2}$
}

The Rho/ROCK/LIMK pathway is central for the mediation of repulsive environmental signals in the central nervous system. Several studies using pharmacological Rho-associated protein kinase (ROCK) inhibitors have shown positive effects on neurite regeneration and suggest additional pro-survival effects in neurons. However, as none of these drugs is completely target specific, it remains unclear how these effects are mediated and whether ROCK is really the most relevant target of the pathway. To answer these questions, we generated adeno-associated viral vectors to specifically downregulate ROCK2 and LIM domain kinase (LIMK)-1 in rat retinal ganglion cells (RGCs) in vitro and in vivo. We show here that specific knockdown of ROCK2 and LIMK1 equally enhanced neurite outgrowth of RGCs on inhibitory substrates and both induced substantial neuronal regeneration over distances of more than $5 \mathrm{~mm}$ after rat optic nerve crush (ONC) in vivo. However, only knockdown of ROCK2 but not LIMK1 increased survival of RGCs after optic nerve axotomy. Moreover, knockdown of ROCK2 attenuated axonal degeneration of the proximal axon after ONC assessed by in vivo live imaging. Mechanistically, we demonstrate here that knockdown of ROCK2 resulted in decreased intraneuronal activity of calpain and caspase 3, whereas levels of pAkt and collapsin response mediator protein 2 and autophagic flux were increased. Taken together, our data characterize ROCK2 as a specific therapeutic target in neurodegenerative diseases and demonstrate new downstream effects of ROCK2 including axonal degeneration, apoptosis and autophagy.

Cell Death and Disease (2014) 5, e1225; doi:10.1038/cddis.2014.191; published online 15 May 2014

Subject Category: Neuroscience

The restorative capacity of the adult mammalian central nervous system (CNS) is severely limited and the inability to re-establish intact axonal projections results in persistent functional deficits following acute traumatic insults to the CNS or in chronic neurodegenerative disorders. Multiple factors hamper the regenerative response of lesioned CNS neurons: degenerative processes, such as neuronal apoptosis and axonal degeneration, lead to the loss of the regenerative substrate, whereas a reduced intrinsic growth state coupled with an inhibitory environment attenuates potential regenerative attempts. ${ }^{1}$

A well-described signaling cascade transmitting inhibitory cues to the growth cone is the RhoA/ROCK/LIMK pathway. ${ }^{2}$ Within this cascade, Rho-associated protein kinase (ROCK) has a key role for the integration of inhibitory signals for axon growth coming from several inhibitory receptors as well as a more recently described function in the regulation of cell survival. $^{3}$ Two isoforms of ROCK exist, ROCK1 (or ROK $\beta$ ) and ROCK2 (or ROK $\alpha$ ). While ROCK1 is mostly expressed in non-neuronal tissue, ROCK2 is strongly expressed in the brain and the spinal cord and its expression increases with age. ${ }^{4,5}$ One of the best characterized downstream targets of
ROCK2 is LIM domain kinase (LIMK). Activation of LIMK results in growth cone collapse. ${ }^{6}$ This effect is mediated via phosphorylation of cofilin, which then becomes inactive and thus unable to severe actin filaments that would be needed to extend the actin-cytoskeleton. ${ }^{7}$ There are two LIMK homologs: LIMK1, which is expressed predominantly in the CNS, and LIMK2, which can be found ubiquitously. ${ }^{8}$

One way of ROCK activation occurs via the trimeric NogoR/ p75/Lingo1 receptor complex, but it can also be mediated by binding of ephrin- $A^{9}$, semaphorin- $3 A^{10}$ and semaphorin-4 $D^{11}$ to their receptors. This contributes not only to the developmental regulation of axon guidance, but can also be of importance in a regenerative setting in the adult mammalian CNS, where developmental guidance cues have been shown to be upregulated following lesion. ${ }^{12}$ Besides inhibition of regeneration, ROCK activity can also be deleterious for cell survival, for example, via activation of phosphatase and tensin homolog deleted on chromosome ten (PTEN) or Fas. ${ }^{13,14}$

Because of these highly relevant functions, inhibition of ROCK activity appears as a promising strategy for neurorestorative approaches. Pharmacological inhibition of ROCK using small-molecule inhibitors, such as the isoquinoline

\footnotetext{
${ }^{1}$ Department of Neurology, University Medicine Göttingen, Göttingen, Germany and ${ }^{2}$ Center for Nanoscale Microscopy and Molecular Physiology of the Brain (CNMPB), Göttingen, Germany

*Corresponding author: JC Koch, Department of Neurology, University Medicine Göttingen, Robert-Koch-Street 40, Göttingen 37075, Germany. Tel: + 49551394749 ; Fax: + 49551 398405; E-mail: jkoch@ @med.uni-goettingen.de

Keywords: ROCK2; LIMK1; axonal degeneration; regeneration; retinal ganglion cells; optic nerve crush

Abbreviations: AAV, adeno-associated viral; AIR, axonal integrity ratio; BDP, breakdown product; CNS, central nervous system; CRMP2, collapsin response mediator protein 2; CSPG, chondroitin sulphate proteoglycan; DIV, day in vitro; GAP43, growth-associated protein 43; LC3, microtubule-associated protein 1 light chain 3; LIMK, LIM domain kinase; mTOR, mammalian target of rapamycin; ONC, optic nerve crush; PTEN, phosphatase and tensin homolog deleted on chromosome ten; RGC, retinal ganglion cell; ROCK, Rho-associated protein kinase; RT-PCR, real-time PCR; siRNA, small interfering RNA; shRNA, small hairpin RNA; TU, transforming unit Received 07.12.13; revised 26.3.14; accepted 28.3.14; Edited by A Verkhratsky
} 
derivative fasudil or the 4-aminopyridine derivative Y-27632, has previously shown to increase axonal regeneration following traumatic lesion. ${ }^{15,16}$ Interestingly, in addition to their pro-regenerative effects, ROCK inhibitors have also shown to act in a neuroprotective manner: different authors, including our group, have demonstrated that inhibition of ROCK increases neuronal survival following MPTP lesion, oxygen-glucose deprivation, axotomy or serum deprivation. ${ }^{16-19}$ However, none of these molecules are specific inhibitors for ROCK and they can inhibit several other kinases and neuronal receptors to a different extent. ${ }^{2,20}$ Pharmacological ROCK inhibitors are even less able to discern between inhibition of ROCK1 and ROCK2, which is of special interest, as different functions for both molecules have been found in the same cell type. ${ }^{21}$ It thus remains unclear, which of the observed effects can be attributed to ROCK2 inhibition and to what extent molecules signaling downstream of ROCK2, such as LIMK1, contribute to the effects mediated by these inhibitors.

In order to more specifically delineate the effects of ROCK2 inhibition, we used here an RNA interference approach using small hairpin RNA (shRNA)-expressing adeno-associated viral (AAV) vectors. ROCK2 and its downstream effector LIMK1 were specifically downregulated in models of cell death, axonal degeneration and regeneration in vitro and in vivo. Our data show that ROCK2 has a major role in the regulation of neuronal survival, axonal stability after lesion and regeneration, whereas inhibition of LIMK1 was only involved in the neurite outgrowth and regeneration. We suggest that the effects of ROCK2 downregulation are mediated by signaling pathways involved in apoptosis, cell survival and autophagy.

\section{Results}

Knockdown of ROCK2 and LIMK1. The most potent and specific small interfering RNAs (siRNA) against ROCK2 and LIMK1 were chosen among several commercially acquired siRNAs after prior testing in neuronal cell cultures. AAV expressing the corresponding shRNA under the control of the $\mathrm{H} 1$ promoter and co-expressing the fluorophore dsRed under the control of the synapsin promoter were generated as described before (for vector map see Figure 1a). ${ }^{22,23}$ For animal experiments, the serotype AAV2 was used, whereas cell culture experiments were performed with the hybrid serotype AAV2/1 consisting of the AAV2 inverted terminal repeats packed into AAV1/AAV2 hybrid capsids (molar ratio $1: 1$ ), because this serotype leads to a more efficient and rapid transduction of retinal ganglion cells (RGCs) in vitro while AAV2 is the most established serotype for retinal transduction in vivo. ${ }^{24,25}$

Effective downregulation of the target proteins ROCK2 and LIMK1 in primary RGCs was confirmed with immunoblot (Figures $1 \mathrm{~b}$ and $\mathrm{c}$ ). Both shRNAs resulted in a significant downregulation of their target protein compared with the control shRNA against EGFP $(n=3$ independent RGC cultures).

To confirm the knockdown specificity, a real-time (RT-) PCR of RNA extracts from primary RGCs was performed. Transduction of RGCs with AAV.LIMK1-shRNA resulted in a
$90 \%$ reduction of normalized expression levels of LIMK1mRNA relative to AAV.EGFP-shRNA, whereas mRNA levels of LIMK2, ROCK1 and ROCK2 were not affected. Correspondingly, AAV.ROCK2-shRNA led to a $75 \%$ reduction of ROCK2-mRNA, whereas levels of LIMK1-, LIMK2- and ROCK1-mRNA were unchanged (Figure 1c).

To test for knockdown efficacy in vivo, the AAV vectors were injected into the vitreous of adult female Wistar rats. Two weeks after intravitreal injection, immunostainings of the retinas showed a clear reduction of the target protein expression levels in the RGCs transduced with AAV expressing the corresponding shRNAs as compared with EGFPshRNA control-treated retinas (Figures 1d and e).

These data thus demonstrate effective knockdown of ROCK2 and LIMK1, respectively, in RGC in vitro and in the rat retina in vivo.

Inhibition of neurite outgrowth is counteracted by downregulation of ROCK2 and LIMK1. To test whether downregulation of the Rho/ROCK/LIMK pathway could counteract environmental inhibitory signaling and thereby increase neurite outgrowth, we cultured RGCs on either laminin or the growth-inhibiting substrate chondroitin sulphate proteoglycan (CSPG), which contains some of the key inhibiting molecules produced by glial cells of the CNS in vivo (major components: neurocan, aggrecan, phosphacan and versican $)^{26}$. RGCs were transduced with different AAVs and the mean neurite length per cell was determined on day in vitro (DIV) 5 (Figure 2; $n=3$ independent RGC cultures; quantification of 10-20 fields of view per group). On laminin, the mean neurite length did not differ significantly between the groups. Compared with untreated control (mean neurite length per cell: $1825 \pm 96 \mu \mathrm{m}$ ) and the control virus AAV.EGFP-shRNA $(2144 \pm 189 \mu \mathrm{m})$, there was, however, a clear trend toward longer neurites in the cells transduced with AAV.ROCK2-shRNA $(2614 \pm 212 \mu \mathrm{m})$ and AAV.LIMK1shRNA $(2530 \pm 224 \mu \mathrm{m})$. On CSPG, untreated RGCs (mean neurite length $626 \pm 58 \mu \mathrm{m}$ ) as well as RGCs transduced with AAV.EGFP-shRNA $(682 \pm 66 \mu \mathrm{m})$ exhibited a significant reduction of neurite outgrowth with the mean neurite length per cell reduced to $35 \%$ compared with laminin. Transduction with AAV.ROCK2-shRNA (mean neurite length $2315 \pm 221 \mu \mathrm{m})$ and AAV.LIMK1-shRNA $(2461 \pm 240 \mu \mathrm{m})$ completely rescued this growth-inhibiting effect of CSPG.

Very moderate cell death can be observed in the primary RGC culture because of in vitro conditions even without addition of neurotoxic substances, and we therefore studied the influence of AAV vectors on neuronal survival. Although AAV vectors expressing EGFP-shRNA and LIMK1-shRNA did not induce any change in cell survival, there was a trend toward a higher number of cells per field of view in cultures transduced with AAV.ROCK2-shRNA (Figure 2d).

Downregulation of ROCK2 and LIMK1 promotes axonal regeneration after optic nerve crush (ONC). To analyze the effects of ROCK2 and LIMK1 downregulation on axonal outgrowth in vivo, AAVs were injected intravitreally in adult female rats. Two weeks later, when sufficient viral expression resulted in stably downregulated target protein levels, a crush lesion of the optic nerve (ONC) was performed as 
a AAV.dsRed.ROCK2-shRNA ITR Sv40-pA Int dsRed Synapsin- H1 ROCK2-shRNA ITR
AAV.dsRed.LIMK1-shRNA [ITR/SV40-pA] [int ds Red Synapsin. H1 LIMK1-shRNA ITR
AAV.dsRed.EGFP-shRNA IITR Sv40-pA|Int dsRed Synapsin. H1 EGFP-shRNA ITR. b
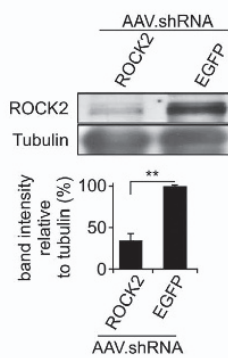

C
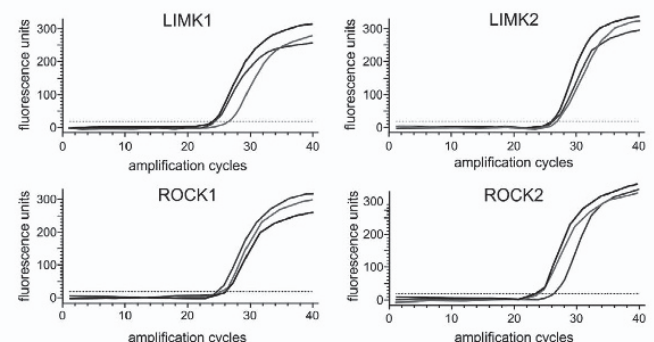

—AAV.EGFP-shRNA $\square$ AAV.ROCK2-shRNA $\square$ AAV.LIMK1-shRNA

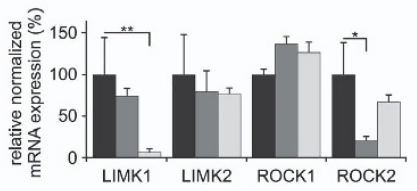

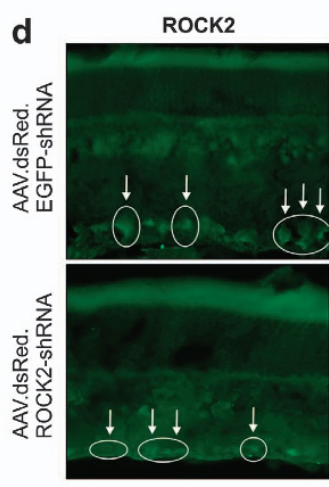
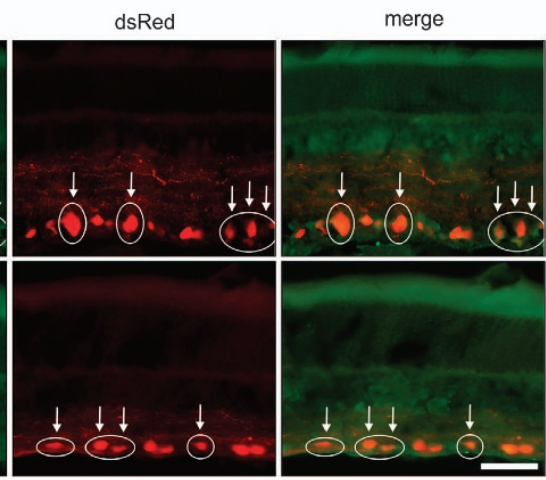

e

LIMK1

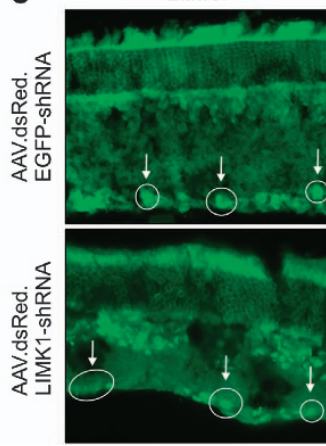

dsRed

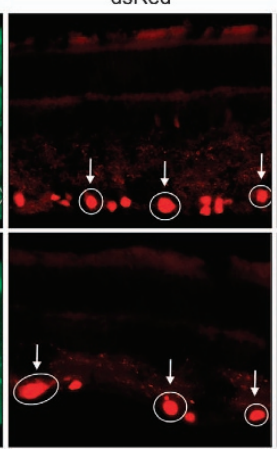

merge

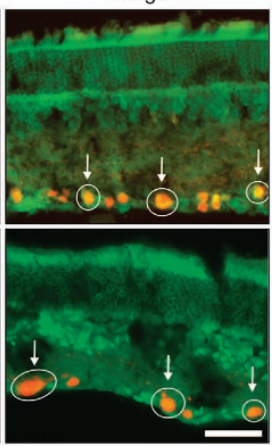

Figure 1 AAV.shRNA-mediated knockdown of ROCK2 and LIMK1 in vitro and in vivo. (a) Vector maps of the AAV used to knockdown ROCK2, LIMK1 and EGFP as control. The respective shRNAs are expressed under the control of an $\mathrm{H} 1$ promoter. The fluorophore dsRed is expressed under the control of a synapsin promoter. ITR, AAV-2 inverted terminal repeat; Int, intron; SV40-pA, SV40 polyadenylation site. (b) Immunoblots for ROCK2 and LIMK1 of primary RGCs transduced with AAV.ROCK2-shRNA and AAV.LIMK1-shRNA compared with AAV.EGFP-shRNA, respectively. The quantifications of the band intensities normalized to loading control show a $>50 \% \mathrm{knockdown}$ efficacy for both AAV-shRNAs ( $n=3$; bars represent means \pm S.E.M. ${ }^{* *} P<0.005$ according to Student's $t$-test). (c) RT-PCR of RNA extracts from primary RGCs transduced with AAV.EGFP-shRNA (black), AAV.ROCK2-shRNA (dark gray) and AAV.LIMK1-shRNA (light gray) on DIV 7. Exemplary amplification results for each primer assessed by SYBR green fluorescence intensities ( $y$ axis) per PCR cycle ( $x$ axis) are shown in the four upper graphs. Quantifications of relative mRNA expression levels normalized to GAPDH mRNA expression are shown at the bottom. (d and e) Immunohistochemistry of transversal retina sections 2 weeks after intravitreal injection of the given AAV. The ROCK2 staining (d; left column) shows a strong signal in the RGCs transduced with AAV.EGFP-shRNA (top row) and reflects the subcellular localization of ROCK2 close to the membrane. In contrast, the RGCs transduced with AAV.ROCK2-shRNA (d; bottom row) show a clearly diminished ROCK2 signal. Correspondingly, the LIMK1 staining (e; left column) shows a stronger signal in the RGCs transduced with AAV.EGFP-shRNA (e; top row) as compared with AAV.LIMK1-shRNA (bottom row). DsRed (middle columns) corresponds to virally transduced RGCs. White arrows highlight representative RGCs transduced with the given AAV (i.e. showing dsRed fluorescence) and their corresponding ROCK2 or LIMK1 staining. Scale bar: $100 \mu \mathrm{m}$

described before. ${ }^{27}$ The animals were killed 4 weeks after ONC and the optic nerves were immunostained with an antibody against growth-associated protein 43 (GAP43) to quantify the number of regenerating axons at increasing distances from the crush site ( $n=10$ optic nerves per group; Figure 3). ${ }^{28}$

In the animals transduced with the control vector (AAV.EGFP-shRNA), only a small number of GAP43-positive neurites had crossed the lesion site and no neurites could be observed at distances of more than $500 \mu \mathrm{m}$ distal from the crush site. In contrast, transduction with AAV.ROCK2-shRNA and AAV.LIMK1-shRNA resulted in a significantly increased number of GAP43-positive neurites in comparison to AAV.EGFP-shRNA at almost all analyzed distances; some neurites could even be followed over distances of $>5000 \mu \mathrm{m}$ distal from the lesion site, which was never observed for AAV.EGFP-shRNA (Figures $3 b$ and c). Although both LIMK1and ROCK2-shRNA equally induced regeneration of single neurites over long distances, only transduction with
AAV.ROCK2-shRNA led to a significantly higher number of shorter neurites adjacent to the crush site (up to $200 \mu \mathrm{m}$ distal from the crush site; Figure 3c). Quantification of the number of GAP43-positive neurites proximal to the crush site showed a significantly increased number in the AAV.ROCK2-shRNAtreated animals as compared with AAV.EGFP-shRNA and AAV.LIMK1-shRNA (Figures 3d and e). A possible explanation for these observations is that AAV.ROCK2-shRNA not only promotes regeneration but might also increase survival of RGCs and/or attenuate axonal degeneration proximal to the lesion site. We therefore followed this hypothesis first in the optic nerve axotomy paradigm.

RGC survival is increased by downregulation of ROCK2 but not LIMK1 after optic nerve axotomy. To further examine possible pro-survival effects of ROCK2- and LIMK1shRNA, an axotomy of the optic nerve was performed 2 weeks after intravitreal AAV injection. For visualization of the RGCs, we injected $2 \mu$ of FluoroGold (5\%) stereotactically in 
a
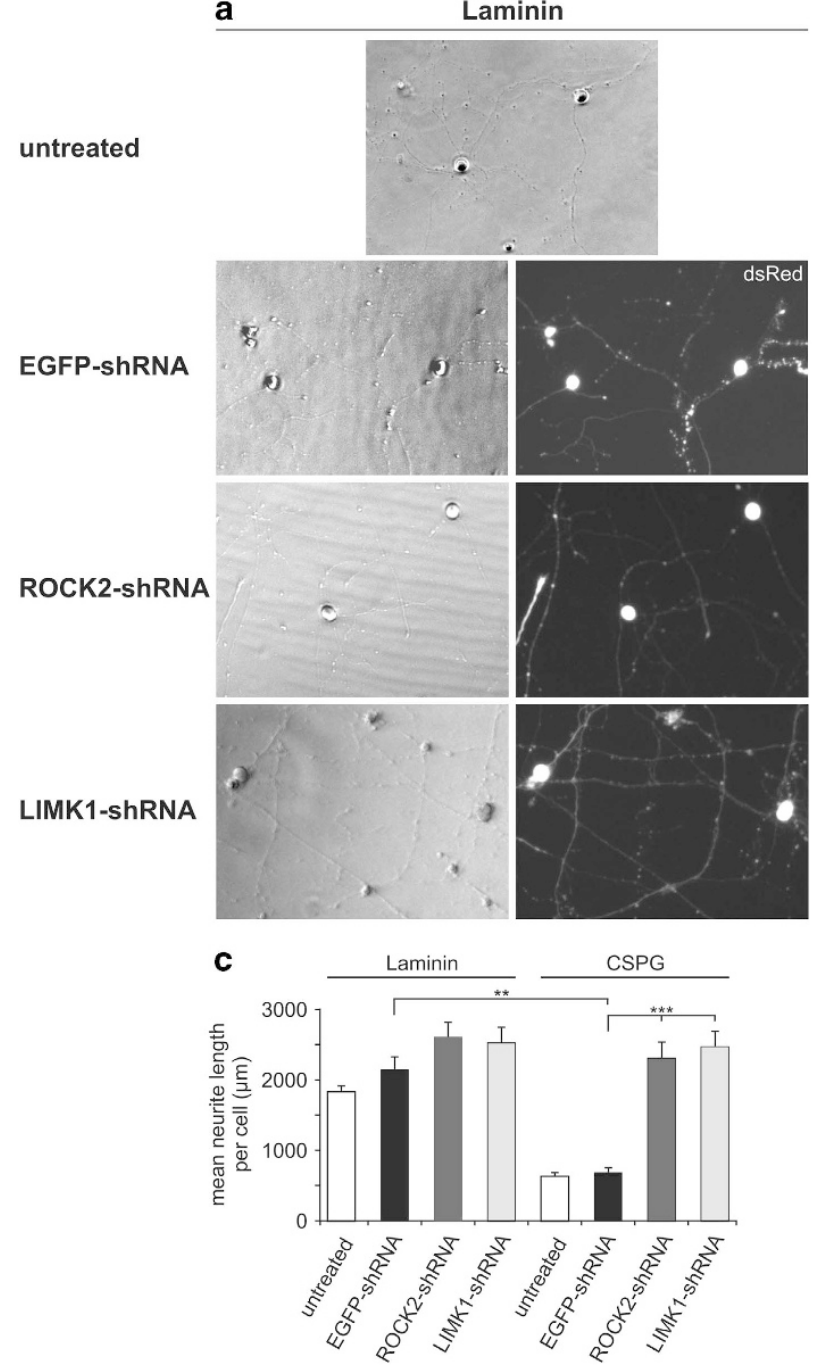

b
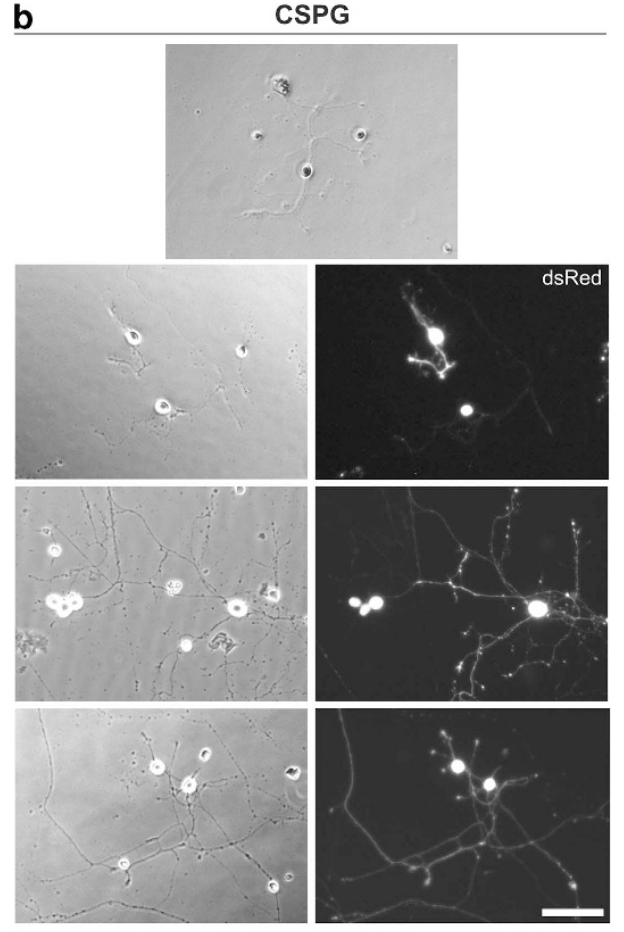

d

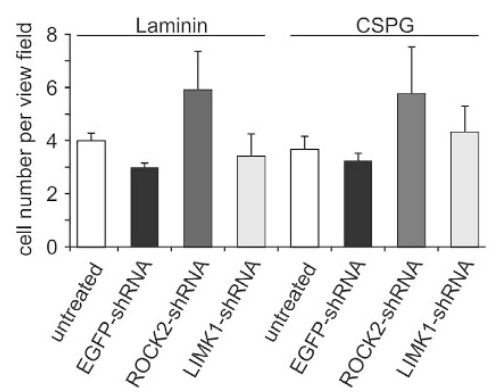

Figure 2 Knockdown of ROCK2 and LIMK1 rescues the inhibitory effects of CSPG on neurite outgrowth in RGCs. (a and b) Representative photos of RGCs transduced with the AAV given on the left side on DIV 5 (phase contrast on the left, dsRed fluorescence on the right representing the virally transduced cells). Neurites of the RGCs transduced with AAV.EGFP-shRNA and without treatment are much shorter on the non-permissive substrate CSPG (b) as compared with Laminin (a). In contrast, RGCs transduced with AAV.ROCK2-shRNA and AAV.LIMK1-shRNA are not affected by CSPG with regards to their neurite growth. Scale bar: $100 \mu \mathrm{m}$. (c) Quantification of the mean neurite length per RGC transduced with the given AAV or untreated and plated either on laminin or CSPG ( $n=3 \mathrm{RGC}$ cultures; bars represent means \pm S.E.M.; ${ }^{* \star} P<0.005$, ${ }^{* * \star} P<0.0005$, according to one-way analysis of variance (ANOVA) followed by Dunnett's post-hoc test). (d) Quantification of the mean cell number per view field of RGCs transduced with the given AAV or untreated and plated either on laminin or CSPG. There was no significant difference between the groups ( $n=3$ RGC cultures; bars represent means \pm S.E.M.)

the colliculus superior on both sides 5 days before the axotomy. FluoroGold is a retrograde neuronal tracer that is taken up by the RGC axons in the colliculus superior and then transported back to the soma in the retina where it labels the RGCs. ${ }^{29}$ Two weeks after axotomy, the number of FluoroGold-labeled RGCs in the central region of the retina was quantified on retina flat-mounts (Figure $4 ; n=5$ retinas per group). Transduction with AAV.ROCK2-shRNA resulted in a significantly increased number of RGCs $(143 \pm 17 \%)$ compared with AAV.EGFP-shRNA, whereas AAV.LIMK1shRNA $(114 \pm 12 \%)$ did not significantly enhance RGC survival.

Axonal degeneration is attenuated by ROCK2 downregulation in vivo. As the downregulation of ROCK2 had a more pronounced effect on the stabilization of axons proximal to the crush site after ONC (3-fold increase compared with control; Figure 3 e) as on RGC survival after axotomy (1.5-fold increase compared to control; Figure 4), we hypothesized that ROCK2 downregulation might have an additional effect on axonal degeneration. To test this hypothesis, we performed an in vivo live imaging of the rat optic nerve following ONC to study axonal degeneration kinetics in the living animal.

A crush lesion of the optic nerve results in the progressive fragmentation of the adjacent $500 \mu \mathrm{m}$ on both sides of the lesion within $6 \mathrm{~h}$ after axonal injury, a process called acute axonal degeneration. ${ }^{27,30,31}$ This process can be analyzed by determining the axonal integrity ratio (AIR) at different time points after ONC. The AIR is defined as the sum length of 
a
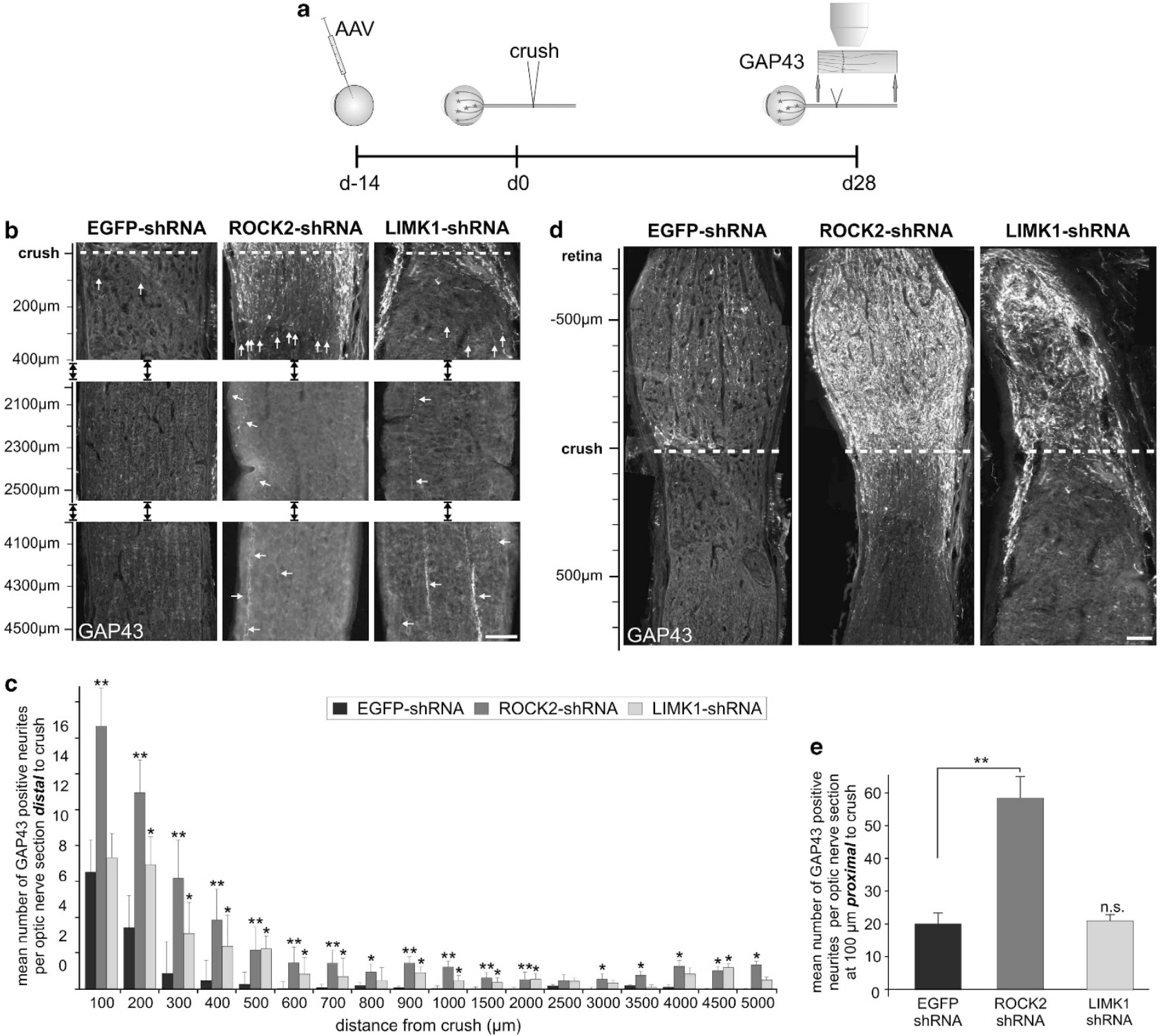

Figure 3 Knockdown of ROCK2 and LIMK1 increases axonal regeneration after optic nerve crush. (a) Schematic drawing of experimental procedures. AAVs were injected intravitreally 14 days before crush lesion of the optic nerve. Twenty-eight days after crush, the animals were killed and the number of GAP43-positive axons was quantified on optic nerve sections at different distances from the crush site. (b) Representative photos of optic nerves transduced with the AAV given on top and immunostained for GAP43 at different distances distal from the crush site (marked on the scale bar at the right side). For AAV.ROCK2-shRNA and AAV.LIMK1-shRNA, single GAP43-positve axons (white arrows) could be followed over distances over $4500 \mu \mathrm{m}$ distal from the crush site. Scale bar: $100 \mu \mathrm{m}$. (c) Quantification of the number of GAP43-positive axons at different distances from the crush site. Transduction with AAV.ROCK2-shRNA and AAV.LIMK1-shRNA significantly increased the number of axons at almost all analyzed positions along the nerve ( $n=10$ optic nerves per group; bars represent means \pm S.E.M.; ${ }^{*} P<0.05 ;{ }^{* *} P<0.005$ according to one-way ANOVA followed by Dunnett's posthoc test). (d) Representative overview photos of the area $500 \mu \mathrm{m}$ adjacent to the crush site of optic nerves transduced with the AAV given on top and immunostained for GAP43. Transduction with AAV.ROCK2-shRNA increased the number of GAP43-positve axons on both sides of the crush. Scale bar: $100 \mu \mathrm{m}$. (e) Quantification of the number of GAP43-positive axons at $100 \mu \mathrm{m}$ proximal to the crush site shows a significant increase only for the optic nerves transduced with AAV.ROCK2-shRNA ( $n=10$ optic nerves per group; bars represent means \pm S.E.M.; ${ }^{* *} P<0.005$, according to one-way ANOVA followed by Dunnett's post-hoc test)

axonal fragments at a given time point divided by the initial total axon length before fragmentation. As axonal disintegration proceeds, the AIR thus decreases. ${ }^{27}$

Two weeks after intravitreal injection of either AAV.ROCK2shRNA or AAV.EGFP-shRNA as control, we performed an in vivo live imaging of the optic nerve over $6 \mathrm{~h}$ following ONC. The AAV-mediated co-expression of the fluorophore dsRed allowed to image specifically the axons that also express the shRNA of interest. On both proximal and distal side of the crush, the adjacent $500 \mu \mathrm{m}$ were monitored and the AIR was calculated every hour from 0 to $6 \mathrm{~h}$ after crush ( $n=8$ proximal and 8 distal axons of four animals per group).

In the animals transduced with AAV.ROCK2-shRNA, axonal degeneration was clearly decelerated on the proximal side of the crush, reflected by a significantly increased AIR compared with AAV.EGFP-shRNA at 4, 5 and $6 \mathrm{~h}$ after crush (Figure 5). The axons in the ROCK2-shRNA group also slowly developed subtle signs of degeneration like axonal swellings 


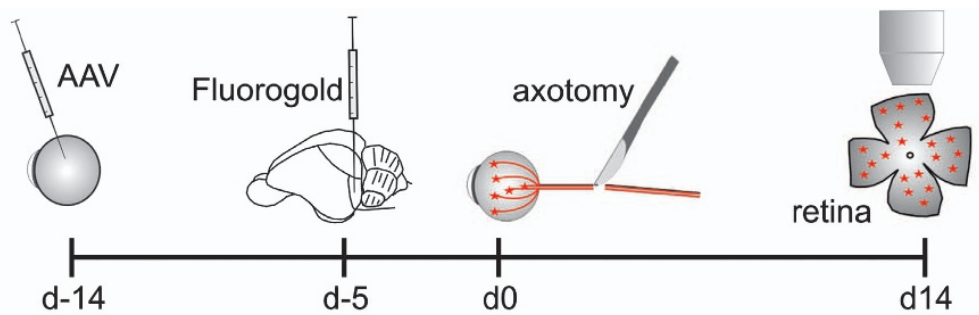

b EGFPShRNA ROCK2shRNA LIMK1shRNA
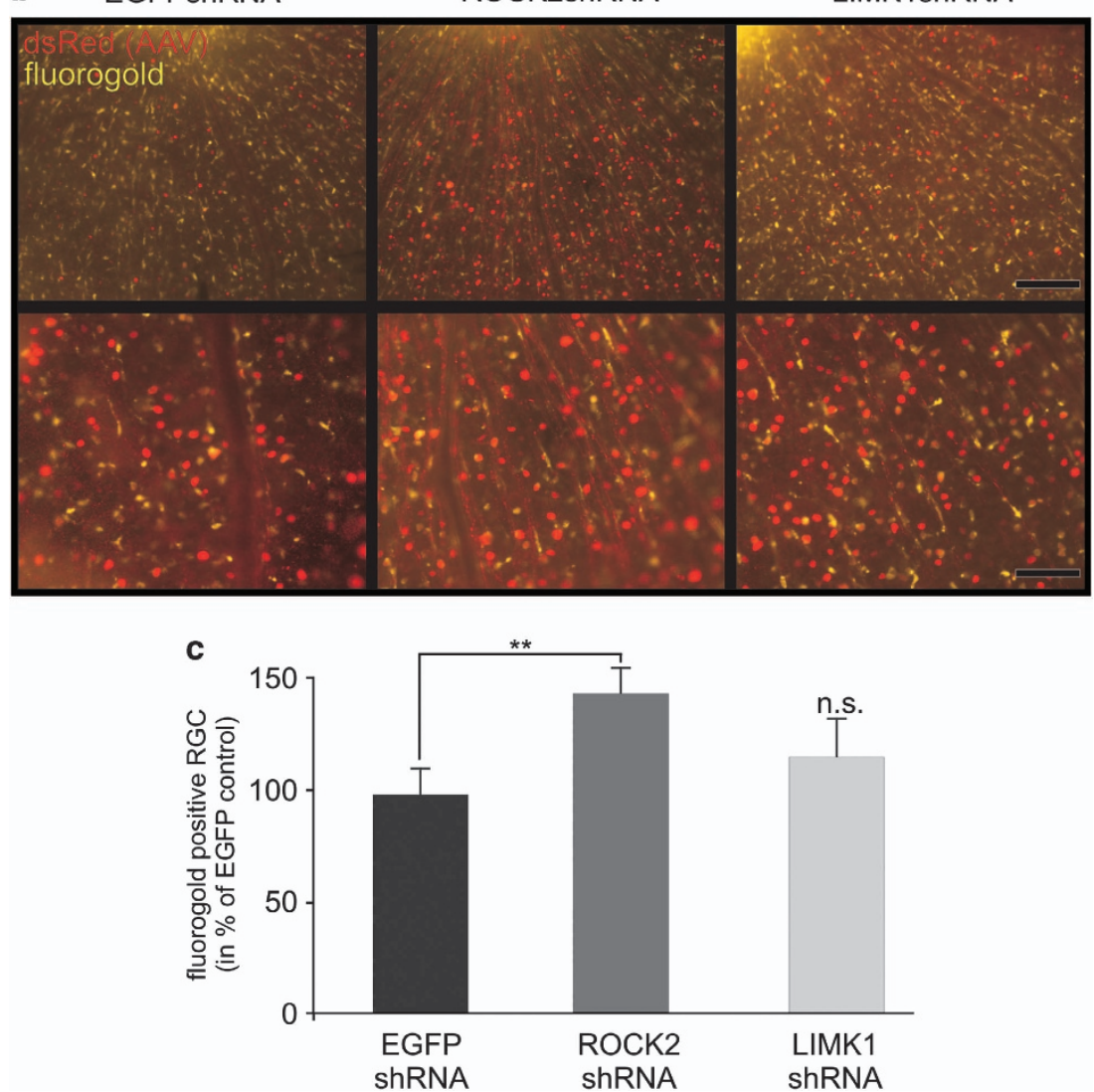

Figure 4 Knockdown of ROCK2 but not LIMK1 increases RGC survival after optic nerve axotomy. (a) Schematic drawing of experimental procedures. AAVs were injected intravitreally 14 days before axotomy. Five days before axotomy, $2 \mu \mathrm{l} 5 \%$ FluoroGold were injected stereotactically in the colliculus superior on both sides in order to label the RGCs. Fourteen days after transection of the optic nerve, the number of retinal ganglion cells was counted on retina flat mounts. (b) Representative photos of the central retina (optic nerve head on top) showing RGCs transduced with the given AAV in red and FluoroGold-positive cells in yellow. Top row: $\times 10$ magnification, scale bar: $400 \mu$ m; bottom row: $\times 20$ magnification, scale bar: $200 \mu \mathrm{m}$. (c) Quantification of the number of FluoroGold-positive RGCs at 2 weeks after axotomy in the central region of the retina (0-1 mm around the optic nerve head) given in percent of AAV.EGFP-shRNA control. Transduction with AAV.ROCK2-shRNA but not AAV.LIMK1-shRNA significantly increased the number of surviving RGCs compared with control ( $n=5$ retinas per group; bars represent means \pm S.E.M.; ${ }^{* \star} P<0.005$; ns: not significant, according to one-way analysis of variance followed by Dunnett's post-hoc test)

(Figure 5c), but did not fragment within the $6 \mathrm{~h}$ time period in contrast to the control axons. On the distal side of the crush, we could also observe some ROCK2-shRNA-transduced axons that degenerated slower as compared with control (Figure $5 \mathrm{c}$ ), but due to a broader variety of degenerative kinetics resulting in larger standard deviations of the AIR, there was no significant difference between AAV.ROCKshRNA and AAV.EGFP-shRNA on the distal side.

ROCK2 downregulation modulates apoptosis, cell survival and autophagy in primary RGCs. To analyze possible molecular downstream targets of ROCK2 and
LIMK1 that could be responsible for the effects described above, we prepared whole-cell protein lysates from primary RGCs on DIV 9 for immunoblot analysis ( $n=3$ independent RGC cultures for every tested protein target). In prior experiments, we examined whether AAV.EGFP-shRNA caused unspecific regulations of tested proteins compared with untreated control or other AAV controls, but could never find such an effect (unpublished).

Calpain has an important role in apoptosis, axonal degeneration and regeneration. ${ }^{32,33}$ Its activity can be assessed by the analysis of the $145-\mathrm{kDa}$ breakdown product (BDP) of $\alpha$ ll-spectrin ( = $\alpha$-fodrin), which is derived specifically 
a

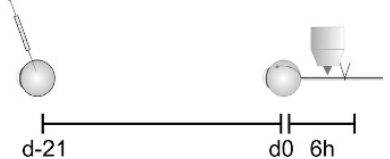

b

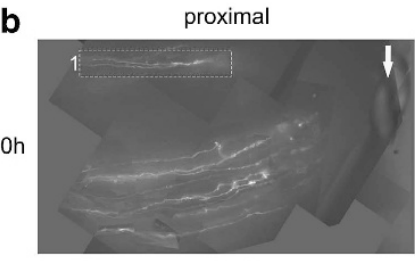

$6 \mathrm{~h}$

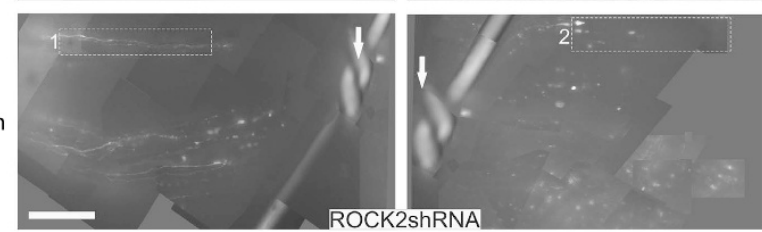

distal

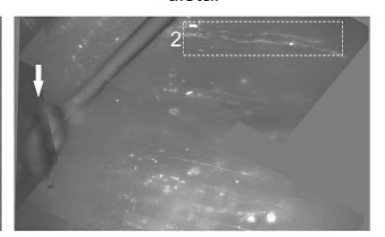

d

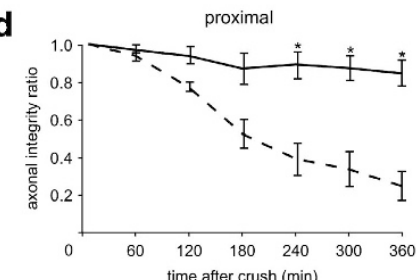

proximal
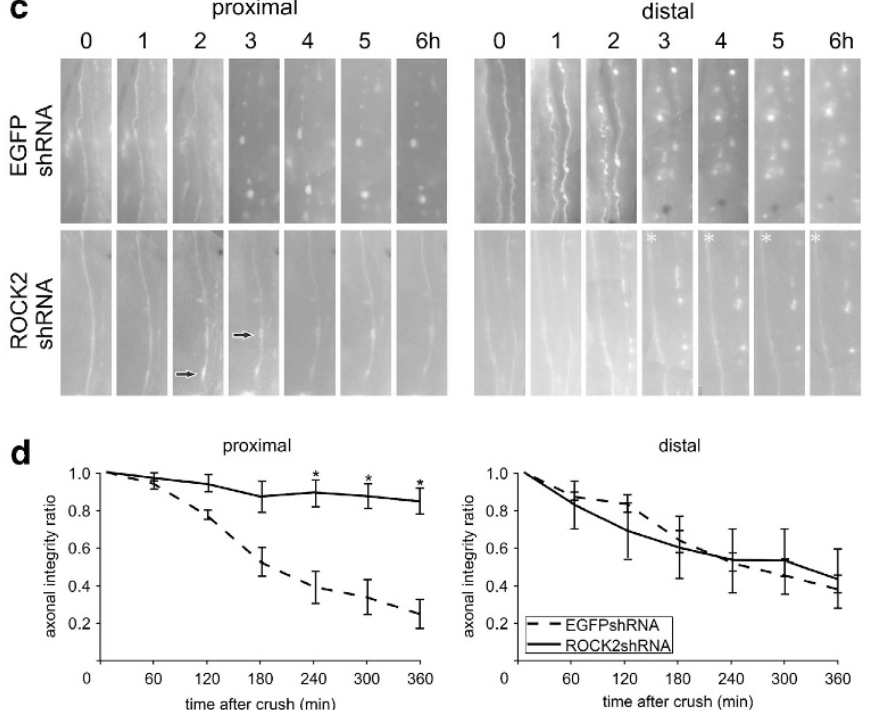

Figure 5 Knockdown of ROCK2 attenuates degeneration of the proximal axon following optic nerve crush assessed by in vivo live imaging. (a) Schematic drawing of experimental procedures. AAVs were intravitreally injected 14-21 days before optic nerve crush. Before and over $6 \mathrm{~h}$ after optic nerve crush an in vivo live imaging of the optic nerve was performed to monitor axonal degeneration in the area $500 \mu \mathrm{m}$ proximal and distal to the crush site. (b) Representative composite overview photos from an in vivo live imaging of an optic nerve transduced with AAV.ROCK2-shRNA at $5 \mathrm{~min}$ (top row, ' $0 \mathrm{~h}$ ') and $6 \mathrm{~h}$ (bottom row, ' $6 \mathrm{~h}$ ') after optic nerve crush. The crush suture (white arrow) can be seen on the right side on the photos from the proximal side of the crush (left column) and on the left side on the photos from the distal side of the crush (right column). A dashed box at both time points highlights two representative axons on each side of the crush. The axons on the proximal side (box ' 1 ') appear stable even at $6 \mathrm{~h}$ after crush, whereas the axons on the distal side (box '2') are already fragmented at this time point. Scale bar: $100 \mu \mathrm{m}$. (c) Representative time row photos of single axons (composite pictures from z-stacks) from an in vivo live imaging of the optic nerve at the time points given on top (in hours after crush). The axons transduced with AAV.EGFP-shRNA (top row) show the typical kinetics of acute axonal degeneration and fragment within $6 \mathrm{~h}$ on both proximal and distal side of the crush. In contrast, axons transduced with AAV.ROCK2-shRNA (bottom row) on the proximal side of the crush show only little signs of degeneration like bulb formation (black arrows) but do not fragment within the $6 \mathrm{~h}$ time period. On the distal side, however, this axon stabilizing effect was not as pronounced as some axons also stayed stable (such as the axon on the left side of the pictures; marked with *), whereas others degenerated with normal kinetics of acute axonal degeneration (such as the axon on the right side). (d) Graphs show the quantification of the axonal integrity ratio (AIR), which is defined as the sum length of axonal fragments divided by the total axon length before fragmentation. On the proximal side, the AIR was significantly higher at later time points in the AAV.ROCK2-shRNA transduced axons reflecting their greater stability. On the distal side, the standard deviations of the AIR in the AAV.ROCK2-shRNA group were large reflecting the varying kinetics of single axons (as depicted in c), but taken together there were no significant differences between the two groups ( $n=8$ axons on both sides of the crush from four optic nerves per group; error bars represent S.E.M.; ${ }^{*} P<0.05$, according to Student's $t$-test)

by calpain cleavage. ${ }^{34}$ Here, RGCs transduced with AAV. ROCK2-shRNA showed significantly lower levels of the 145kDa BDP of $\alpha$ ll-spectrin compared with AAV.EGFP-shRNA and AAV.LIMK1-shRNA (Figures $6 a$ and $b$ ). This suggests a lower calpain activity in the RGC transduced with AAV. ROCK2-shRNA.

Interestingly, AAV.ROCK2-shRNA reduced not only the levels of the calpain-specific 145-kDa BDP of $\alpha$ ll-spectrin but also resulted in a significant decrease of the 120-kDa BDP compared with AAV.EGFP-shRNA and AAV.LIMK1-shRNA (Figures $6 a$ and $b$ ). The 120-kDa BDP of $\alpha$ ll-spectrin is derived from cleavage by caspase-3, a major protease essential for the execution of apoptosis. ${ }^{34}$ To prove that the activity of caspase- 3 is reduced after downregulation of ROCK2, we further performed a western blot analysis against cleaved (i.e., active) caspase-3 (Figures $6 \mathrm{c}$ and d). ${ }^{35}$ This also showed significantly reduced levels of cleaved caspase- 3 in the RGCs transduced with AAV.ROCK2-shRNA compared with AAV.EGFP-shRNA and AAV.LIMK1-shRNA.

Next, expression levels of the pro-survival factors pAkt and $\mathrm{Bcl} 2$ were evaluated (Figures $6 \mathrm{e}$ and f). We observed an increased expression of the pro-survival factor phospho-Akt (pAkt; Ser473) after downregulation of ROCK2, which is in line with previous results in other models. ${ }^{17}$ The expression of Bcl2 was not significantly altered.
Next, we checked expression levels of phospho-PTEN, as PTEN is not only an important upstream regulator of Akt but also a proposed target of ROCK2. ${ }^{36}$ In our culture model, its expression levels were not affected by AAV.ROCK2-shRNA (Figures $6 \mathrm{~g}$ and $\mathrm{h}$ ).

Collapsin response mediator protein 2 (CRMP2) has a crucial role in axon outgrowth and polarity through promoting microtubule assembly in the developing CNS. ${ }^{37}$ ROCK2 has been shown to phosphorylate and thereby inactivate CRMP2. ${ }^{38}$ Interestingly, we found a substantial increase of the expression levels of both CRMP2 splicing variants CRMP2A and CRMP2B in the ROCK2-shRNA transduced RGCs as compared with controls (Figures $6 g$ and $h$ ).

Surprisingly, downregulation of ROCK2 resulted in a strong regulation of the autophagy pathway (Figures $6 \mathrm{i}$ and $\mathrm{j}$ ). To assess autophagy, we analyzed the expression levels of the two isoforms of microtubule-associated protein 1 light chain 3 (LC3): LC3-I (18 kDa) and its PE-conjugated form LC3-II $(16 \mathrm{kDa})$. For evaluation of autophagic flux, the cells were treated with the vacuolar-type $\mathrm{H}^{+}$-ATPase inhibitor bafilomy$\operatorname{cin} A 1$, which arrests autophagy at the lysosomal level reliably unmasking the transit of LC3-II through the autophagic pathway. $^{39}$

All conditions showed similar expression levels of LC3-I. For LC3-II, we found a significant increase in the RGCs 

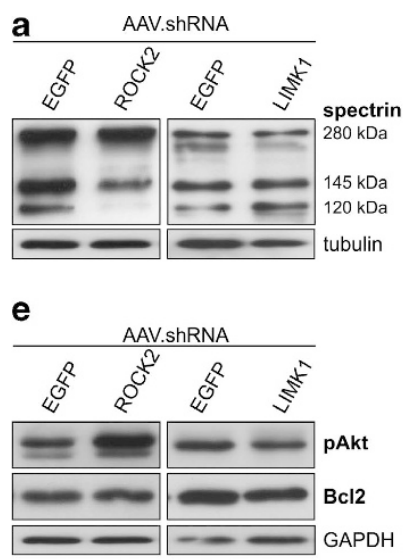

b

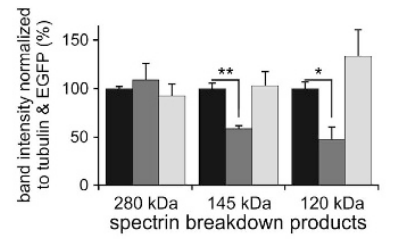

f

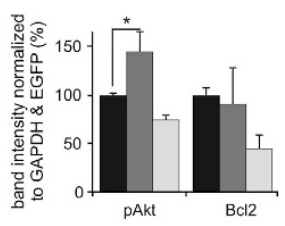

c
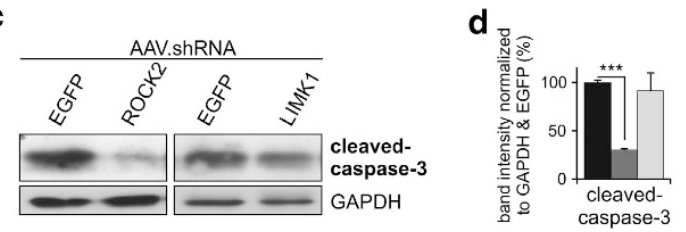

i

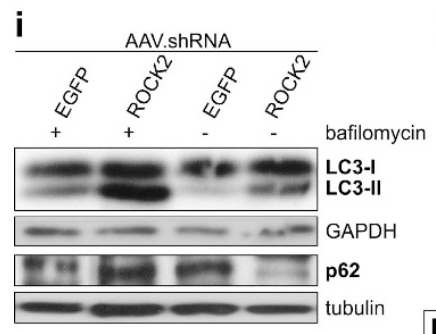

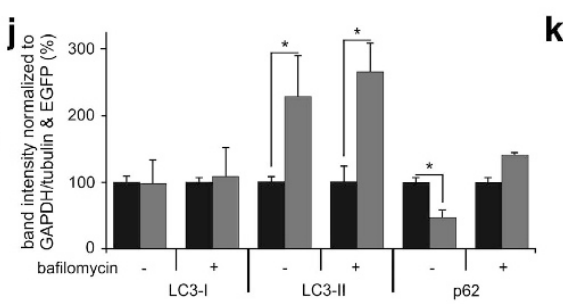

EGFP-shRNA — ROCK2-shRNA $\square$ LIMK1-shRNA

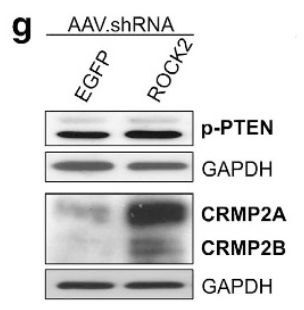

k

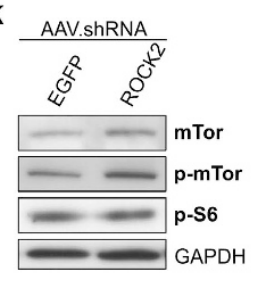

h

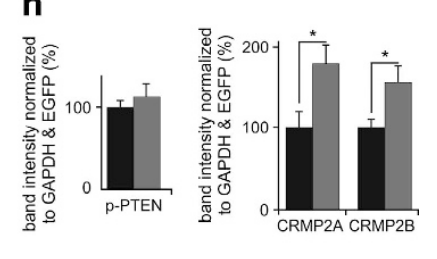

Figure 6 ROCK2 downregulation modulates apoptosis, cell survival and autophagy. Immunoblotting was performed of protein lysates from primary RGC cultures on DIV 9. Photos of representative western blots are shown on the left side, the quantification of band intensities normalized to tubulin or GAPDH as loading control and relative to AAV.EGFP-shRNA (EGFP) are depicted on the right side ( $n=3$ independent RGC cultures; bars represent means \pm S.E.M.; ${ }^{*} P<0.05$, ${ }^{\star *} P<0.005$, ${ }^{\star \star *} P<0.0005$, according to one-way analysis of variance followed by Dunnett's post-hoc test). ( $\mathbf{a}$ and $\mathbf{b}$ ) Calpain activity (corresponding to the $145 \mathrm{kDa}$ breakdown product (BDP) of spectrin) as well as caspase-3 activity (corresponding to the $120 \mathrm{kDa}$ BDP of spectrin) are significantly decreased in RGCs transduced with AAV.ROCK2-shRNA (ROCK2) compared with AAV.EGFP-shRNA (EGFP) and AAV.LIMK1-shRNA (LIMK1). (c and d) Decreased caspase-3 activity in the RGCs transduced with AAV.ROCK2-shRNA was confirmed by immunoblotting for cleaved caspase-3. (e and f) Detection of the pro-survival factors pAkt and Bcl2 showed increased pAkt levels in the RGCs transduced with AAV.ROCK2-shRNA, whereas Bcl2 levels did not differ significantly among the groups. ( $\mathbf{g}$ and $\mathbf{h}$ ) Levels of phospho-PTEN were not altered in the RGCs transduced with AAV.ROCK2-shRNA. However, protein levels of the two CRMP2 splicing variants CRMP2A and CRMP2B were almost doubled in the ROCK2-shRNA group as compared with EGFP-shRNA control. (i and j) ROCK2-shRNA increased LC3-II levels by more than two-fold compared with EGFP-shRNA, whereas p62-levels were decreased. Addition of $10 \mathrm{nM}$ bafilomycin for $4 \mathrm{~h}$ before lysis further enhanced LC3-II levels and this effect was stronger in the ROCK2-shRNA group as compared with control. (k and I) Expression levels of the main upstream regulator of autophagy mTOR, its phosphorylated form p-mTOR and the downstream target p-S6 were not significantly changed by AAV.ROCK2shRNA expression

transduced with AAV.ROCK2-shRNA compared with AAV.EGFP-shRNA. Bafilomycin treatment (10 $\mathrm{nM}$ for $4 \mathrm{~h}$ ) further increased levels of LC3-II. This increase was also more pronounced for AAV.ROCK2-shRNA than for AAV.EGFP-shRNA, thus suggesting an increased autophagic flux in the RGCs transduced with AAV.ROCK2-shRNA.

To confirm these results, we analyzed the levels of the protein SQSTM1/p62 (p62). P62 serves as a link between LC3 and ubiquitinated substrates. ${ }^{40}$ We found significantly decreased p62 levels after ROCK2shRNA transduction (Figures $6 i$ and $j$ ) reaffirming its induction of autophagic flux.

One of the most important upstream regulators of autophagy is the mammalian target of rapamycin (mTOR), which integrates growth factor, nutrient and energy signaling. Activation of mTOR results in an inhibition of autophagy. Activity of mTOR complex 1 can be monitored by following the phosphorylation of its substrate ribosomal protein S6 (p-S6). ${ }^{41}$ As ROCK2-shRNA did not change the expression levels of mTOR, phospho-mTOR or p-S6 (Ser 235/236) (Figures 6k and I), its autophagy-inducing effects are likely to be mediated by mTOR-independent mechanisms.

\section{Discussion}

In this study, we addressed specifically ROCK2-mediated effects on cell survival, axonal regeneration and degeneration, and identified novel signaling mechanisms regulated by ROCK2. Based on our present results, three major biological effects can be specifically attributed to the knockdown of ROCK2: inhibition of apoptosis, attenuation of axonal degeneration and promotion of neurite outgrowth (Figure 7).

The role of the Rho/ROCK/LIMK pathway in neurite outgrowth has been addressed in numerous studies, ${ }^{3}$ but the specific contribution of single members of this cascade remained insufficiently understood. RhoA inhibition with C3-toxin was shown to induce neuronal differentiation and neurite outgrowth in vitro and axonal regeneration in vivo. ${ }^{42-44}$ ROCK inhibition, using different pharmacological inhibitors, including fasudil and Y-27632, promoted axonal regeneration and neurite outgrowth not only in RGC in vitro and in traumatic lesion models in the optic nerve and spinal cord in vivo, but also in models of neurodegenerative diseases. ${ }^{15,16,17,45,46}$ So far, only limited data on LIMK1 inhibition exists: in rat cortical neurons, downregulation of LIMK1 was shown to 


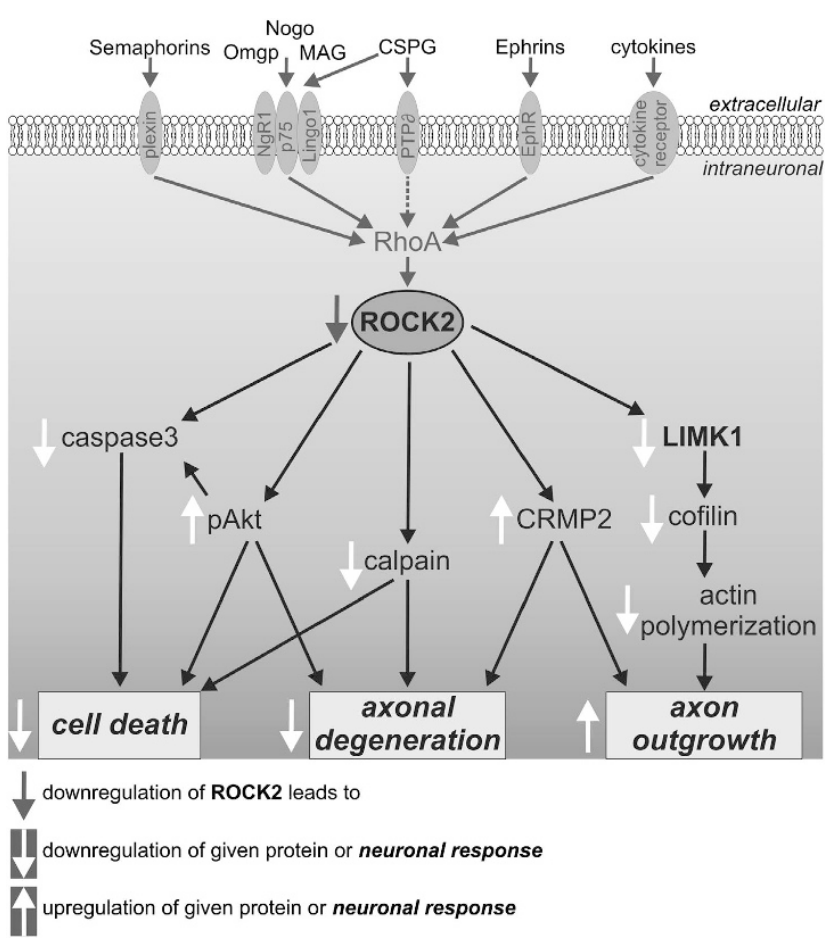

Figure 7 Schematic drawing of the effects mediated by ROCK2 downregulation observed in the present study. For details refer to the Discussion

have a crucial role in BDNF/CaMKIlb-mediated neurite outgrowth. ${ }^{47}$

We show here that specific downregulation of ROCK2 in RGCs is sufficient to promote neurite outgrowth to a similar extent as reported before for the more unspecific ROCK inhibitors. This places ROCK2 at center stage as regulator of neurite growth and argues for the development and therapeutic evaluation of more specific ROCK2 inhibitors. As downregulation of ROCK2 and LIMK1 was equally effective in inducing neurite outgrowth of RGCs on CSPG and longdistance regeneration after $\mathrm{ONC}$, it seems plausible that the specific neurite growth-promoting effect of ROCK inhibition is mainly mediated via LIMK1 and its downstream modulation of cofilin and actin. ${ }^{7}$ These findings indicate that LIMK1 might be an interesting therapeutic target for selective regulation of neurite outgrowth.

In line with previous observations, we show that ROCK2 downregulation increased cell survival of RGC in vivo and that this effect was accompanied by the activation of Akt. ${ }^{18,48,49}$ In addition, we show that ROCK2 downregulation results in a decreased caspase-3 activity. Recently, application of the pharmacological ROCK inhibitor fasudil in a rat cerebral ischemia model was shown to inhibit caspase-3 activity and to decrease infarct area. ${ }^{50}$ Our findings thus further extend the spectrum of specific ROCK2-mediated effects to anti-apoptotic signaling, which could additionally contribute to the neuroprotective effects seen in vivo. A possible link between ROCK2 and the apoptosis pathway might be calpain. We found that ROCK2 downregulation leads to decreased calpain activity in RGCs. Calpain can induce apoptosis, at least partly via caspase-3 activation. ${ }^{51}$ Concurrent activation of Rho/ROCK and calpain has been shown before to occur in growth cone collapse, ${ }^{52}$ but the direct connection between the two pathways remains to be discovered.

Interestingly, we found an upregulation of autophagic flux in RGCs as a result of ROCK2 downregulation. So far, only little is known about the effects of ROCK2 on autophagy. Although one study reported increased macroautophagy in a cell line after treatment with the ROCK-inhibitor Y-27632, ${ }^{53}$ another study reported that ROCK inhibition impaired the starvationmediated autophagic response. ${ }^{54}$ The increased autophagic flux caused by ROCK inhibition might be helpful under certain stress conditions including neuronal degeneration or aggregate formation and might therefore help to explain the effectiveness of ROCK inhibition under these circumstances.

The attenuation of axonal degeneration caused by downregulation of ROCK2 has not been described before. It has, however, been reported that pharmacological ROCK inhibition leads to reduced axon retraction after axotomy in chicken dorsal root ganglion cells in vitro, at least partly via myosin. ${ }^{55}$ Interestingly, a recent screening series including quantitative proteomic screens and in silico analyses revealed ROCK2 as one of six potential main regulators of synaptic and axonal degeneration in vivo. ${ }^{56}$

In axonal degeneration, calcium influx and subsequent calpain activation have been implicated as the initial events on top of a multifactorial molecular cascade leading to the fragmentation of the axon. ${ }^{30,31}$ As we demonstrate here, ROCK2 downregulation substantially decreases calpain activity and this might partially explain the positive effects on axonal degeneration. Besides, the direct effects of ROCK2 downregulation on the actin cytoskeleton as explained above might contribute to the protective effects on axon degeneration.

Moreover, we found substantially increased expression levels of CRMP2 under ROCK2 knockdown conditions. CRMP2 has an important role in inhibiting axonal degeneration and is also crucial for axonal outgrowth, axonal transport and growth cone mobility. ${ }^{57}$ ROCK2 can phosphorylate and thereby inactivate CRMP2, but the finding of increased CRMP2 levels after ROCK2 downregulation was surprising. A possible explanation might be a slower degradation of phosphorylated CRMP2 when ROCK2 levels are decreased.

Taken together, we show here that the downregulation of ROCK2 and LIMK1 both lead to increased neurite outgrowth in different experimental paradigms, whereas only downregulation of ROCK2 increased survival of lesioned neurons. Moreover, downregulation of ROCK2 attenuated acute axonal degeneration. Interestingly, ROCK2 downregulation increased autophagic flux and decreased calpain and caspase 3 activity. These data extend previous studies with pharmacological ROCK inhibitors and specify ROCK2 as a central therapeutic target for traumatic and degenerative nervous system disorders. ${ }^{15,16,17}$

\section{Materials and Methods}

Cloning and production of AAV. Among a pool of four purchased siRNAs against ROCK2 and LIMK1 (Qiagen, Hilden, Germany), the most effective siRNAs were chosen based on prior testing in cell culture. The sequences of these siRNAs were cloned in the vector pSUPER-hSyn-EGFP-CytBAS (GenBank ID: AY640629) as described before. ${ }^{23,58}$ In brief, $200 \mathrm{pmol}$ of the forward and reverse primers containing the shRNA sequence were dissolved in $25 \mu \mathrm{l}$ water and $25 \mu \mathrm{l}$ of $2 \times$ annealing buffer $(20 \mathrm{mM}$ Tris, $\mathrm{pH} 7.8,100 \mathrm{mM} \mathrm{NaCl}$ and $0.2 \mathrm{mM}$ EDTA). The solution was boiled $5 \mathrm{~min}$ and then cooled to room 
temperature. Annealed oligos $(200 \mathrm{fmol})$ were ligated to $22.5 \mathrm{fmol}$ of the BgI2/Hind3-cut backbone fragment of pSUPER-hSyn-EGFP-CytB-AS.

The sequences of the shRNA primers were as follows (in bold: sequence of the respective siRNA-sense and -antisense strand, in italics: sequence of the hairpin turn):

ROCK2-shRNA forward primer: $5^{\prime}$-GATCCCCTGCAAAGTTTATTATGATATAC TTCCTGTCATATATCATAATAAACTTTGCATTTTTGGAAA-3';

ROCK2-shRNA reverse primer: 5'-AGCTTTTCCAAAAATGCAAAGTTTATTAT

GATATATGACAGGAAGTATATCATAATAAACTTTGCAGGG-3';

LIMK1-shRNA forward primer: 5'-GATCCCCAACACCTAGGGACGTATTAAAC TTCCTGTCATTTAATACGTCCCTAGGTGTTTTTTTGGAAA-3',

LIMK1-shRNA reverse primer: 5'-AGCTTTTCCAAAAAAACACCTAGGGACGT ATTAAATGACAGGAAGTTTAATACGTCCCTAGGTGTTGGG-3'

This first cloning step resulted in the vectors pSUPER-hSyn-EGFP-H1-ROCK2shRNA and pSUPER-hSyn-EGFP-H1-LIMK1-shRNA. From these vectors, the insert containing the $\mathrm{H} 1$-promoter and the shRNA were cut out with a BstB1/Hind3 digestion and cloned in a BstB1/Hind3-cut pAAV-9(5)hSyn-DsRed2N1-CytbASohneNot (GenBank ID: AY640633) resulting in the vectors pAAV-9(5)hSyn-DsRedH1-ROCK2-shRNA and pAAV-9(5)hSyn-DsRed-H1-LIMK1-shRNA. These vectors were used for AAV production. Cloning of the control vector PAAV-9(5)hSynDsRed-H1-EGFP-shRNA was described before (=pAAV-Si-I (GenBank ID: AY6406334)). ${ }^{58}$ All plasmids were sequenced to confirm their correct identity.

Production of AAV was performed as reported before. ${ }^{22}$ Briefly, 293-HEK cells were transfected with calcium phosphate, HEPES-buffered saline and a serotypespecific plasmid mix (for AAV2: pAAV-RC, pHELPER (both from Stratagene, La Jolla, CA, USA) and the respective PAAV expression vector in a 1:1:1 molar ratio; for AAV2/1: pAAV-RC, pH21, pHELPER and the respective pAAV expression vectors in a $0.5: 0.5: 1: 1$ molar ratio). The $\mathrm{pH} 21$ vector, expressing AAV serotype 1 capsids, was a gift from Helen Fitzsimons (Neurologix, Inc. OSU Comprehensive Cancer Center, Columbus, OH, USA) and Matthew During (Molecular Virology, Immunology and Medical Genetics, Columbus, $\mathrm{OH}, \mathrm{USA}$ ). Forty-eight hours after transfection, cells were harvested and AAV were purified by dialysis and virus gradient centrifugation in iodixanol. Fast protein liquid chromatography was performed to obtain high titer viral stocks. The virus stocks were then tested on primary cortical neurons for transduction efficacy and toxicity and viral titers were determined using $\mathrm{qPCR}$.

Primary rat RGC culture, evaluation of neurite outgrowth, immunoblotting, RT-PCR. RGCs were prepared from 7-day-old Wistar rat pups and enriched by a two-step panning protocol for Thy-1 to $99.5 \%$ purity. ${ }^{59}$ Cells were plated in 24-well plates with a density of 4000 cells per well for neurite outgrowth experiments and 50000 cells per well for protein lysates. Per well $500 \mu \mathrm{l}$ RGC medium were added composed of serum-free neurobasal medium (Gibco, distributed by Life Technologies, Darmstadt, Germany) supplemented with sodium-pyruvate (Sigma-Aldrich, Seelze, Germany), glutamine, N-acetyl-cysteine, triiodothyronine, Sato (bovine serum albumin, transferrin, progesterone, putrescine, sodium selenite; Gibco), forskolin (final concentration $10 \mathrm{mM}$ ), human BDNF (final concentration $50 \mathrm{ng} / \mathrm{ml}$; Tebu, Offenbach, Germany), insulin (final concentration $5 \mathrm{mg} / \mathrm{ml}$; Sigma-Aldrich), CNTF (final concentration $10 \mathrm{ng} / \mathrm{ml}$; Tebu) and B27 supplement. Coverslips (Sarstedt, Nümbrecht, Germany) were coated with poly-o-lysine (Sigma-Aldrich) and either laminin $(20 \mu \mathrm{g} / \mathrm{ml}$; Sigma-Aldrich) or CSPG $(50 \mu \mathrm{g} / \mathrm{ml}$, Chemicon, Darmstadt, Germany; major components: neurocan, aggrecan, phosphacan and versican; for neurite outgrowth experiments only).

At $4 \mathrm{~h}$ after seeding, the medium was reduced to $250 \mu \mathrm{l}$ per well and virus was added $\left(0.5 \times 10^{8}\right.$ transforming units (TUs) of AAV2/1 per well). Twenty-four hours later, $250 \mu$ l fresh medium were added to each well. Transduction efficacy of RGCs ( $>90 \%$ after DIV 3 ) and possible virus toxicity ( $<10 \%$ cell death until DIV 5 ) were checked regularly.

For neurite outgrowth experiments, photos of four randomly chosen visual fields per well were taken at an inverted microscope (Axiovert, Zeiss, Göttingen, Germany) on DIV 5. On the photos, the total neurite length per view field was quantified using the neurite tracing module of the ImageJ plugin Neuron ${ }^{60}$ and then divided by the number of neurons per view field to obtain the mean neurite length per cell. Results from three independent experiments were statistically evaluated using one-way analysis of variance followed by Dunnett's post-hoc test with significance at $P<0.05$ and standard error of mean (S.E.M.)

For western blot analyses, RGCs were lysed in ice-cold lysis buffer $(10 \mathrm{mM}$ HEPES, $142 \mathrm{mM} \mathrm{KCl}, 5 \mathrm{mM} \mathrm{MgCl}, 2.1 \mathrm{mM}$ EGTA, IGEPAL, protease and phosphatase inhibitor and dithiothreitol) on DIV 9. For analysis of autophagic flux,
$10 \mathrm{nM}$ Bafilomycin (Sigma-Aldrich) was added to the medium $4 \mathrm{~h}$ before lysis in selected conditions. After ultrasound sonification, protein lysates were resolved on SDS-PAGE and blotted on a polyvinylidene difluoride or nitrocellulose membrane. For further standard western blot processing, the following antibodies were used: monoclonal antibodies against $\alpha$ ll-spectrin (Enzo, Lörrach, Germany); Bcl2 (Santa Cruz, Heidelberg, Germany), GAPDH (Biotrend, Köln, Germany), LC3 (NanoTools, Teningen, Germany), mTOR (Millipore, Darmstadt, Germany) and phospho-mTOR (Cell Signaling, Leiden, Netherlands); polyclonal antibodies against pAkt (Ser473), cleaved caspase-3, CRMP2, LIMK1, phospho-PTEN, phosphor-S6 Ribosomal protein (Ser235/236; all Cell Signaling), p62 (Sigma-Aldrich), ROCK2 (Santa Cruz) and tubulin (Abcam/Epitomics, Berlin, Germany); secondary antibodies: horseradish peroxidase-coupled IgG (Dianova, Hamburg, Germany). For visualization enhanced chemiluscence (ECL-solution: $250 \mathrm{mM}$ Luminol, $90 \mathrm{mM} \mathrm{p}$-coumaric acid, $1 \mathrm{M}$ Tris $/ \mathrm{HCl}, 30 \%$ hydrogen peroxide) was used. Blots were analyzed with Image $J$ software (open freeware provided by the $\mathrm{NIH}$, Bethesda, MD, USA; http:// imagej.nih.gov/ij/). Statistical analyses from three independent experiments were performed using paired Student's t-test (mean \pm S.E.M.).

For RT-PCR, RNA was isolated from AAV-treated RGCs on DIV 7. Cells were lysed with TRIzol reagent. After addition of 1-bromo-3-chlor-propane, cell lysates were centrifuged for $15 \mathrm{~min}$. The upper aqueous layer was extracted, mixed with isopropanol and stored at $-20^{\circ} \mathrm{C}$ overnight. After another centrifugation, the RNA pellet was washed with $75 \%$ ethanol several times. The pellet was dried and then dissolved in $\mathrm{H}_{2} \mathrm{O}$. RNA was quantified using a Nanodrop spectrophotometer (Thermo Scientific, Wilmington, DE, USA). The RNA extracts were transcribed into cDNA using the QuantiTect reverse transcription kit (Qiagen) according to the manufacture's instructions. RT-PCR of the CDNA samples was performed using QuantiTect SYBR green PCR kits (Qiagen) in a C1000 Touch Thermal Cycler (Bio-Rad, Hercules, CA, USA). The following primers were used (all from Qiagen): GAPDH: Rn_Gapd_1_SG (NM_017008) (QT00199633), LIMK1: Rn_Limk1_1_SG (NM_031727) (QT00180621), LIMK2: Rn_Limk2_1_SG (NM_024135) (QT00177842), ROCK1: Rn_RGD:620424_1_SG (NM_031098) (QT00194579), ROCK2: Rn_Rock2_1_SG (NM_013022) (QT00192808; all as QuantiTect primer assays). Analysis and data evaluation were performed with the Bio-Rad CFX manager software (Bio-Rad). Relative mRNA expression levels were normalized to the respective GAPDH mRNA expression levels.

Animal experiments. Animals were treated according to the regulations of the local animal research council and legislation of the State of Lower Saxony, Germany. For all experiments, adult female Wistar rats (200-300 g, Charles River, Sulzfeld, Germany) were used. All procedures (intravitreal virus injection, ONC, stereotactical injection, axotomy, optic nerve live imaging) were performed under deep anesthesia with $10 \%$ ketamine $(95 \mathrm{mg} / \mathrm{kg}$ body weight) and $2 \%$ xylazine (7 mg/kg body weight) injected intraperitoneally.

Intravitreal virus injection, ONC and GAP43 immunohistochemistry. AAV2 $(1 \times 10$ e9 TU in $5 \mu$ PBS) was injected intravitreally with a Hamilton syringe as described before (Hamilton, Bonaduz, Switzerland). ${ }^{27}$

Two weeks after intravitreal injections, an ONC was performed as described previously. ${ }^{27}$ In brief, the orbit was incised along the orbital rim and the lacrimal gland was moved to the front. The eye bulb was slightly rotated by pulling the superior rectus muscle. After removing the retro-orbital connecting tissue and longitudinally incising the dura, the optic nerve was exposed. For the crush lesion, a 10-0 polyamide suture (Ethicon, Somerville, NJ, USA) was constricted around the optic nerve at a distance of $2 \mathrm{~mm}$ from its insertion into the eye bulb for a duration of $30 \mathrm{~s}$. Then the suture was removed and all tissue put back in situ.

Twenty-eight days after ONC, animals were perfused transcardially $(250 \mathrm{~m}$ phosphate-buffered saline followed by $200 \mathrm{ml} 4 \%$ paraformaldehyde in phosphatebuffered saline (pH 7.4). The optic nerve and eye bulb were removed en bloc and post-fixed in $4 \%$ paraformaldehyde for $1 \mathrm{~h}$. The retina was flat-mounted in $30 \%$ glycerol and examined for viral transduction efficacy (20-60\% of all RGCs). The optic nerves were incubated in $30 \%$ sucrose for $48 \mathrm{~h}$ and then cryosectioned. Longitudinal sections $(16 \mu \mathrm{m})$ were stained with GAP43 (Abcam monoclonal antibody) and secondary Cy2-labeled antibodies (Dianova), counter-stained with 4,6-diamidino-2phenylindole (Sigma) and mounted in Moviol (Hoechst, Frankfurt, Germany).

Optic nerve sections were photographed with an Axioplan microscope (Zeiss) equipped with AxioVision Software (Zeiss). The number of GAP43-positive axons was counted at defined distances from the crush site. For statistical analysis, the numbers of axons at a given distance from the crush site were compared with AAV.EGFP-shRNA using the Student's t-test with standard error of mean (S.E.M.). 
Stereotactical injections and optic nerve axotomy. To visualize RGC for axotomy experiments, we performed stereotactical FluoroGold injections into the colliculus superior at 9 days after intravitreal AAV injection $\left(1 \times 10^{9}\right.$ TU AAV2 $\left.(5 \mu \mathrm{l})\right)$. The skull was exposed with a midline incision and two small holes were drilled $6.5 \mathrm{~mm}$ posterior to bregma and $1.8 \mathrm{~mm}$ lateral to the midline on both sides. $2 \mu \mathrm{l} 5 \%$ FluoroGold (Biotrend) were stereotactically injected using a self-made glass pipette attached to a micromanipulator (Kopf, Tujunga, CA, USA). The needle was inserted $3.2 \mathrm{~mm}$ into the brain injecting at a speed of $500 \mu \mathrm{l} / \mathrm{min}$ over $2 \mathrm{~min}$. The exposed skull was closed and rats allowed to wake up from anesthesia.

Five days later (14 days after intravitreal AAV injection) the optic nerve was exposed as described above for the ONC. It was then transected at $2 \mathrm{~mm}$ from the posterior eye pole taking special care not to damage the retinal blood supply.

On day 14 post-axotomy, animals were perfused and the retinas flat-mounted as described above for ONC. FluoroGold-labeled RGCs were counted under an Axioplan microscope (Zeiss) using a UV filter $(365 / 420 \mathrm{~nm})$ per visual field in the central region of the retina $(0-1 \mathrm{~mm}$ from the optic nerve head along the retina diameter; six visual fields per retina). RGCs were discriminated from other FluoroGold-positive cells (microglia, macrophages etc.) by morphological criteria (soma size, form of processes).

Optic nerve live imaging. Live imaging of the optic nerve was performed 14-21 days after intravitreal AAV injection $\left(1 \times 10^{9}\right.$ TU AAV2 $\left.(5 \mu l)\right)$ as reported before. ${ }^{27}$ In brief, surgery and ONC were performed as described above. Before ONC, the rat was transferred to an Axioplan microscope (Zeiss) adapted for live imaging. Fluorescent pictures were taken of the area $500 \mu \mathrm{m}$ proximal and distal to the crush site before and $5 \mathrm{~min}$ after the crush and then every hour until $6 \mathrm{~h}$ after crush. Special care was taken to give optimal anesthesia and life support to the animal (constant usage of warming pad, measurement of heart rate and oxygen saturation, fluid substitution).

Within the original z-stack pictures, single axons were extracted and projected to one plane using the function 'extended depth of focus' of the axiovision software (Zeiss) and manual corrections. For each axon, the AIR was calculated at all time points. The AIR is defined as the sum length of axonal fragments divided by the total axon length before fragmentation. The AIRs at a given time point were statistically compared between the AAV.ROCK2-shRNA and the AAV.EGFP-shRNA groups using a Student's t-test.

\section{Conflict of Interest}

The authors declare no conflict of interest.

Acknowledgements. We thank Anna-Elisa Roser and Lars Tatenhorst for helping to establish the RT-PCR. JCK and PL were supported by a grant of the Deutsche Forschungsgemeinschaft (LI 1308/3-1). MB and PL were supported by the Cluster of Excellence and DFG Research Center Nanoscale Microscopy and Molecular Physiology of the Brain (CNMPB), Göttingen.

\section{Author contributions}

Experiments were performed by JCK and EB. AAV were cloned and produced by JCK and UM. JCK, LT, MB and PL conceived the experiments. JCK and PL wrote the manuscript.

1. Liu K, Tedeschi A, Park KK, He Z. Neuronal intrinsic mechanisms of axon regeneration. Annu Rev Neurosci 2011; 34: 131-152.

2. Mueller BK, Mack $\mathrm{H}$, Teusch $\mathrm{N}$. Rho kinase, a promising drug target for neurological disorders. Nat Rev Drug Discov 2005; 4: 387-398.

3. Tonges L, Koch JC, Bahr M, Lingor P. ROCKing regeneration: Rho kinase inhibition as molecular target for neurorestoration. Front Mol Neurosci 2011; 4: 39.

4. Hashimoto R, Nakamura Y, Kosako H, Amano M, Kaibuchi K, Inagaki M et al. Distribution of Rho-kinase in the bovine brain. Biochem Biophys Res Commun 1999; 263: 575-579.

5. Duffy P, Schmandke A, Schmandke A, Sigworth J, Narumiya S, Cafferty WB et al. Rho-associated kinase II (ROCKII) limits axonal growth after trauma within the adult mouse spinal cord. J Neurosci 2009; 29: 15266-15276.

6. Endo M, Ohashi K, Sasaki Y, Goshima Y, Niwa R, Uemura T et al. Control of growth cone motility and morphology by LIM kinase and Slingshot via phosphorylation and dephosphorylation of cofilin. J Neurosci 2003; 23: 2527-2537.
7. Maekawa M, Ishizaki T, Boku S, Watanabe N, Fujita A, Iwamatsu A et al. Signaling from Rho to the actin cytoskeleton through protein kinases ROCK and LIM-kinase. Science 1999; 285: 895-898.

8. Mori T, Okano I, Mizuno K, Tohyama M, Wanaka A. Comparison of tissue distribution of two novel serine/threonine kinase genes containing the LIM motif (LIMK-1 and LIMK-2) in the developing rat. Brain Res Mol Brain Res 1997; 45: 247-254.

9. Shamah SM, Lin MZ, Goldberg JL, Estrach S, Sahin M, Hu L et al. EphA receptors regulate growth cone dynamics through the novel guanine nucleotide exchange factor ephexin. Cell 2001; 105: 233-244.

10. Dontchev VD, Letourneau PC. Growth cones integrate signaling from multiple guidance cues. J Histochem Cytochem 2003; 51: 435-444.

11. Lin X, Ogiya M, Takahara M, Yamaguchi W, Furuyama T, Tanaka H et al. Sema4D-plexinB1 implicated in regulation of dendritic spine density through RhoA/ROCK pathway. Neurosci Lett 2007; 428: 1-6.

12. Moreau-Fauvarque C, Kumanogoh A, Camand E, Jaillard C, Barbin G, Boquet I et al. The transmembrane semaphorin Sema4D/CD100, an inhibitor of axonal growth, is expressed on oligodendrocytes and upregulated after CNS lesion. J Neurosci 2003; 23: 9229-9239.

13. Zhang L, Valdez JM, Zhang B, Wei L, Chang J, Xin L. ROCK inhibitor Y-27632 suppresses dissociation-induced apoptosis of murine prostate stem/progenitor cells and increases their cloning efficiency. PLoS One 2011; 6: e18271.

14. Piazzolla D, Meissl K, Kucerova L, Rubiolo C, Baccarini M. Raf-1 sets the threshold of Fas sensitivity by modulating Rok-alpha signaling. J Cell Biol 2005; 171: 1013-1022.

15. Lingor $\mathrm{P}$, Teusch $\mathrm{N}$, Schwarz K, Mueller R, Mack H, Bahr M et al. Inhibition of Rho kinase (ROCK) increases neurite outgrowth on chondroitin sulphate proteoglycan in vitro and axonal regeneration in the adult optic nerve in vivo. J Neurochem 2007; 103: 181-189.

16. Lingor P, Tonges L, Pieper N, Bermel C, Barski E, Planchamp V et al. ROCK inhibition and CNTF interact on intrinsic signalling pathways and differentially regulate survival and regeneration in retinal ganglion cells. Brain 2008; 131(Pt 1): 250-263.

17. Tonges L, Frank T, Tatenhorst L, Saal KA, Koch JC, Szego EM et al. Inhibition of rho kinase enhances survival of dopaminergic neurons and attenuates axonal loss in a mouse model of Parkinson's disease. Brain 2012; 135(Pt 11): 3355-3370.

18. Yamashita K, Kotani Y, Nakajima Y, Shimazawa M, Yoshimura S, Nakashima S et al. Fasudil, a Rho kinase (ROCK) inhibitor, protects against ischemic neuronal damage in vitro and in vivo by acting directly on neurons. Brain Res 2007; 1154: 215-224.

19. Tura A, Schuettauf F, Monnier PP, Bartz-Schmidt KU, Henke-Fahle S. Efficacy of Rho-kinase inhibition in promoting cell survival and reducing reactive gliosis in the rodent retina. Invest Ophthalmol Visual Sci 2009; 50: 452-461.

20. Davies SP, Reddy H, Caivano M, Cohen P. Specificity and mechanism of action of some commonly used protein kinase inhibitors. Biochem J 2000; 351: 95-105.

21. Shi J, Wu X, Surma M, Vemula S, Zhang L, Yang Y et al. Distinct roles for ROCK1 and ROCK2 in the regulation of cell detachment. Cell Death Dis 2013; 4: e483.

22. Zolotukhin S, Byrne BJ, Mason E, Zolotukhin I, Potter M, Chesnut K et al. Recombinant adeno-associated virus purification using novel methods improves infectious titer and yield. Gene Therapy 1999; 6: 973-985.

23. Koch JC, Barski E, Lingor P, Bahr M, Michel U. Plasmids containing NRSE/RE1 sites enhance neurite outgrowth of retinal ganglion cells via sequestration of REST independent of NRSE dsRNA expression. FEBS J 2011; 278: 3472-3483.

24. Dinculescu A, Glushakova L, Min SH, Hauswirth WW. Adeno-associated virus-vectored gene therapy for retinal disease. Hum Gene Therapy 2005; 16: 649-663.

25. Koch JC, Solis GP, Bodrikov V, Michel U, Haralampieva D, Shypitsyna A et al. Upregulation of reggie-1/flotillin-2 promotes axon regeneration in the rat optic nerve in vivo and neurite growth in vitro. Neurobiol Dis 2013; 51: 168-176.

26. Niederost BP, Zimmermann DR, Schwab ME, Bandtlow CE. Bovine CNS myelin contains neurite growth-inhibitory activity associated with chondroitin sulfate proteoglycans. J Neurosci 1999; 19: 8979-8989.

27. Koch JC, Knoferle J, Tonges L, Michel U, Bahr M, Lingor P. Imaging of rat optic nerve axons in vivo. Nat Protoc 2011; 6: 1887-1896.

28. Schaden $\mathrm{H}$, Stuermer CA, Bahr M. GAP-43 immunoreactivity and axon regeneration in retinal ganglion cells of the rat. $J$ Neurobiol 1994; 25: 1570-1578.

29. Berkelaar M, Clarke DB, Wang YC, Bray GM, Aguayo AJ. Axotomy results in delayed death and apoptosis of retinal ganglion cells in adult rats. J Neurosci 1994; 14 : 4368-4374.

30. Kerschensteiner M, Schwab ME, Lichtman JW, Misgeld T. In vivo imaging of axonal degeneration and regeneration in the injured spinal cord. Nat Med 2005; 11: 572-577.

31. Knoferle J, Koch JC, Ostendorf T, Michel U, Planchamp V, Vutova P et al. Mechanisms of acute axonal degeneration in the optic nerve in vivo. Proc Natl Acad Sci USA 2010; 107: $6064-6069$

32. Gitler D, Spira ME. Real time imaging of calcium-induced localized proteolytic activity after axotomy and its relation to growth cone formation. Neuron 1998; 20: 1123-1135.

33. Ma M. Role of calpains in the injury-induced dysfunction and degeneration of the mammalian axon. Neurobiol Dis 2013; 60C: 61-79.

34. Wang KK. Calpain and caspase: can you tell the difference? Trends Neurosci 2000; 23 20-26.

35. Polverino AJ, Patterson SD. Selective activation of caspases during apoptotic induction in HL-60 cells. Effects Of a tetrapeptide inhibitor. J Biol Chem 1997; 272: 7013-7021.

36. Li Z, Dong X, Wang Z, Liu W, Deng N, Ding Y et al. Regulation of PTEN by Rho small GTPases. Nat Cell Biol 2005; 7: 399-404. 
37. Yoshimura T, Kawano Y, Arimura N, Kawabata S, Kikuchi A, Kaibuchi K. GSK-3beta regulates phosphorylation of CRMP-2 and neuronal polarity. Cell 2005; 120: 137-149.

38. Arimura N, Inagaki N, Chihara K, Menager C, Nakamura N, Amano $\mathrm{M}$ et al. Phosphorylation of collapsin response mediator protein-2 by Rho-kinase. Evidence for two separate signaling pathways for growth cone collapse. J Biol Chem 2000; 275 23973-23980.

39. Klionsky DJ, Abdalla FC, Abeliovich H, Abraham RT, Acevedo-Arozena A, Adeli K et al. Guidelines for the use and interpretation of assays for monitoring autophagy. Autophagy 2012; 8: 445-544.

40. Bjorkoy G, Lamark T, Brech A, Outzen H, Perander M, Overvatn A et al. p62/SQSTM1 forms protein aggregates degraded by autophagy and has a protective effect on huntingtininduced cell death. J Cell Biol 2005; 171: 603-614.

41. Brunn GJ, Hudson CC, Sekulic A, Williams JM, Hosoi H, Houghton PJ et al. Phosphorylation of the translational repressor PHAS-I by the mammalian target of rapamycin. Science 1997; 277: 99-101.

42. Kozma R, Sarner S, Ahmed S, Lim L. Rho family GTPases and neuronal growth cone remodelling: relationship between increased complexity induced by $\mathrm{Cdc} 42 \mathrm{Hs}$, Rac1, and acetylcholine and collapse induced by RhoA and lysophosphatidic acid. Mol Cell Biol 1997; 17: 1201-1211.

43. Gu H, Yu SP, Gutekunst CA, Gross RE, Wei L. Inhibition of the Rho signaling pathway improves neurite outgrowth and neuronal differentiation of mouse neural stem cells. Int $J$ Physiol Pathophysiol Pharmacol 2013; 5: 11-20.

44. Boato F, Hendrix S, Huelsenbeck SC, Hofmann F, Grosse G, Djalali S et al. C3 peptide enhances recovery from spinal cord injury by improved regenerative growth of descending fiber tracts. J Cell Sci 2010; 123(Pt 10): 1652-1662.

45. Wu BQ, Bi ZG, Qi Q. Inactivation of the Rho-ROCK signaling pathway to promote neurologic recovery after spinal cord injuries in rats. Chinese Med J 2013; 126: 3723-3727.

46. Ahmed Z, Berry M, Logan A. ROCK inhibition promotes adult retinal ganglion cell neurite outgrowth only in the presence of growth promoting factors. Mol Cell Neurosci 2009; 42: 128-133.

47. Saito A, Miyajima K, Akatsuka J, Kondo H, Mashiko T, Kiuchi T et al. CaMKIllbeta-mediated LIM-kinase activation plays a crucial role in BDNF-induced neuritogenesis. GenesCells 2013; 18: 533-543.

48. Jeon BT, Jeong EA, Park SY, Son H, Shin HJ, Lee DH et al. The Rho-kinase (ROCK) inhibitor Y-27632 protects against excitotoxicity-induced neuronal death in vivo and in vitro. Neurotox Res 2013; 23: 238-248.

49. Hirata A, Inatani M, Inomata $\mathrm{Y}$, Yonemura N, Kawaji T, Honjo M et al. Y-27632, a Rho-associated protein kinase inhibitor, attenuates neuronal cell death after transient retinal ischemia. Graefe's archive for clinical and experimental ophthalmology $=$ Albrecht von Graefes Archiv fur klinische und experimentelle Ophthalmologie 2008; 246: 51-59.

50. Wu J, Li J, Hu H, Liu P, Fang Y, Wu D. Rho-kinase inhibitor, fasudil, prevents neuronal apoptosis via the Akt activation and PTEN inactivation in the ischemic penumbra of rat brain. Cell Mol Neurobiol 2012; 32: 1187-1197.
51. Sharma AK, Rohrer B. Calcium-induced calpain mediates apoptosis via caspase- 3 in a mouse photoreceptor cell line. J Biol Chem 2004; 279: 35564-35572.

52. To KC, Church J, O'Connor TP. Growth cone collapse stimulated by both calpain- and Rhomediated pathways. Neuroscience 2008; 153: 645-653.

53. Bauer PO, Wong HK, Oyama F, Goswami A, Okuno M, Kino $Y$ et al. Inhibition of Rho kinases enhances the degradation of mutant huntingtin. J Biol Chem 2009; 284: 13153-13164.

54. Aguilera MO, Beron W, Colombo MI. The actin cytoskeleton participates in the early events of autophagosome formation upon starvation induced autophagy. Autophagy 2012; 8: 1590-1603.

55. Gallo G. Myosin II activity is required for severing-induced axon retraction in vitro. Exp Neurol 2004; 189: 112-121.

56. Wishart TM, Rooney TM, Lamont DJ, Wright AK, Morton AJ, Jackson M et al. Combining comparative proteomics and molecular genetics uncovers regulators of synaptic and axonal stability and degeneration in vivo. PLoS Genet 2012; 8: e1002936.

57. Khanna R, Wilson SM, Brittain JM, Weimer J, Sultana R, Butterfield A et al. Opening Pandora's jar: a primer on the putative roles of CRMP2 in a panoply of neurodegenerative, sensory and motor neuron, and central disorders. Future Neurol 2012; 7: 749-771.

58. Michel U, Malik I, Ebert S, Bahr M, Kugler S. Long-term in vivo and in vitro AAV-2-mediated RNA interference in rat retinal ganglion cells and cultured primary neurons. Biochem Biophys Res Commun 2005; 326: 307-312.

59. Barres BA, Silverstein BE, Corey DP, Chun LL. Immunological, morphological, and electrophysiological variation among retinal ganglion cells purified by panning. Neuron 1988; 1: 791-803.

60. Meijering E, Jacob M, Sarria JC, Steiner P, Hirling H, Unser M. Design and validation of a tool for neurite tracing and analysis in fluorescence microscopy images. Cytometry A 2004; 58: $167-176$.

(c) (i) (2)(2) Cell Death and Disease is an open-access journal BY ${ }^{2}$ published by Nature Publishing Group. This work is licensed under a Creative Commons Attribution-NonCommercialShareAlike 3.0 Unported License. The images or other third party material in this article are included in the article's Creative Commons license, unless indicated otherwise in the credit line; if the material is not included under the Creative Commons license, users will need to obtain permission from the license holder to reproduce the material. To view a copy of this license, visit http://creativecommons.org/licenses/ by-nc-sa/3.0/ 